\title{
Quantification of Epicardial and Thoracic Adipose Tissue using WOA Optimized CNN
}

\author{
N.Sangeetha A.Kathirvel, P.Indira Priya, R.Latha
}

\begin{abstract}
Cardiac fat depots are associated with the heart diseases. Epicardial fat and thoracic fat plays the major role in the development of cardiovascular disease. The increased thickness of the epicardial and thoracic fat leads to several diseases such as metabolic syndrome, coronary atherosclerosis, etc. It is necessary to quantify the epicardial adipose tissue and thoracic adipose tissue. There are different imaging and assessing techniques for epicardial and thoracic adipose tissue quantification. These tissues can be quantified automatically or manually from the CT and MRI cardiac scans. The quantification of the epicardial fat and thoracic fat requires segmentation of these fats by various segmentation methods and then they are quantified. This project proposes the fully automatic segmentation and quantification of the epicardial and thoracic adipose tissues from the cardiac CT scan images using the krill herd optimization algorithm and fuzzy c-means segmentation algorithm. The whale optimization algorithm performs the feature selection process. The fuzzy $c$ means algorithm is used for the segmentation process by means of clustering which segments the epicardial fat and paracardial adipose tissue $(E A T \& P A T)$ from the input image. The segmented epicardial and paracardial fat region are then used for the quantification process which provides the epicardial and thoracic fat volume. The thoracic fat is the combination of the epicardial and paracardial fat. This proposed system is implemented by using the MATLAB code. The proposed system is simple, fully automatic and produces accurate results.
\end{abstract}

Keywords-Fuzzy K-means algorithm; Epicardial Adipose Tissue (EAT); Paracardial Adipose Tissue (PAT);

\section{INTRODUCTION}

Obesity is known as a major cardiac disease threat factor (Visscher et al. 2001; Hubert et al. 1983; Manson et al. 1990). In comparison to the subcutanic fat, which is placed under the skin, the quantification of visceral fat (fat covering the inner viscera) shows an excellent correlation with the growth of atherosclerotic conditions and metabolic syndrome parameters. Consequently, certain techniques were created to quantify the non-invasive fat composition, in specific evaluating the complete body fat and visceral tissue (Jeong et al. 2007).

Visceral abdominal tissue is the fat used to quantify visceral material. The technique for this quantification by CT is described by Borkan (Borkan et al. 1982). The epicardial fat is the accumulation of visceral fluid which is present in the cardiovascular and interventricular spaces lining the coronary arteries of the epicardial (Manson et al., 1990). However, latest information indicates its contribution

N.Sangeetha A.Kathirvel, Department of Computer Science and Engineering, MNM JAIN Engineering college, Chennai, Tamilnadu, India.

P.Indira Priya, Professor and HOD, Department of Computer Science and Engineering, MNM JAIN Engineering college, Chennai, Tamilnadu, India.

R.Latha, Professor, Department of Computer Science and Engineering, MNM JAIN Engineering college, Chennai, Tamilnadu, India.
Revised Manuscript Received on July 18, 2019.

to the growth of coronary atheroscle rosis (Iacobellis et al., 2005) are less well established about the real function and metabolic pathophysiology of epicardial fat. Numerous cytokines and atherosclerosis proteins are produced in the epicardial bone (Mazurek, et al., 2003). In combination with the fact that the tissues are not anatomically blocked with the coronary arteries, this indicates that the vessels have a proatherogenic impact.

The CT was used for long periods of time to quantify visceral fat accumulation. This assessment is done with the abdominal CT in a single cut. In view of the latest advances in CT and its growing implementation in the heart region, the quantification of fat deposition in the core is becoming increasingly interesting. As shown by Gorter et al. (Gorter et al. 2008) some quantification methods have been used. But these procedures are usually manual and require a considerable time after treatment. Trying to automate this task has been successful and, as Dey describes, pericardial fat can be quantified automatically (Dey et al. 2008). These techniques, however, assess the heart's total fat without differentiating between epicardium. Because of the clinical interest in epicardial fat measurement and due to the absence of a method that enables its automatic quantification, this document presents a method that can in certain cases automatically quantify epicardial fat while in others only needs a minimal user interference.

\section{FAT QUANTIFICATION METHOD}

The method of automating epicardial fat assessment has been split into two components. First, the image will be preprocessed for the removal of information that could mislead pericardium identification, while second portion will involve pericardium segmentation and subsequent fat quantification.

In this research the images under consideration were first developed using Dey's (Dey et al. 2008) technique, which extracts apart from the heart all other structures. The preprocessing phase in an algorithm is focused on the reality that distinct tissues have distinct Hounsfield Units (HU) a linear transformation of each tissue's initial linear attenuation ratio (Borkan et al. 1982). For fat, the range between -30 and $-190 \mathrm{HU}$ is regarded (Dey et al. 2008). A regionally increasing method for the detection of cardiac muscle; limit for removing elevated pressure marks, because they do not constitute muscle; and a median filter to minimize noise are the measures used for the pre-processing algorithm. 
This patch of fat also prevents the removal of the pericardium line. Figure 1c indicates the picture after the cardiac muscle has been removed. This image still contains artifacts that can be separated from the fat and the pericardial line via a threshold filter of greater intensity. Figure 1d demonstrates the final outcome after the threshold and a medium noise attenuation filter has been applied.

\section{RELATED WORK}

Conci et al. suggest a technique consisting of fully automatically segmenting two kinds of cardiac adipose tissues that are distinguished by CT image pericardium by means of the performance acquisition protocol used to optimize and improve earlier established 2D fat segmentation depending on pre-knowledge of epicardial structure and subjective and objective metric correspondence are used for coronary metal rating Lazar Velicki et al. Using the 2D segmentation technique, IoannisA et al. provided a hierarchy, multiple-feature, fuzzy affinity-based tissue segmentation computing framework in medical images. An assessment of our methodology's precision shows bias and consensus boundaries comparable to inter-observer variability intrinsic in conventional segmentation.

A new method to automatic quantification of EAT was introduced by Angel Torrado-Carvajal et al, with "a priori" anatomical data. A region of interest (ROI) was obtained from the final-diastolic heart phase, accompanied by the GVF snake algorithm SzymonŽukasik1 and others compared with other heuristic techniques and more traditionally, the KHA formula used for the learning of the artificial neural network (ANN). For identification tasks, the predicted ANN learning method was confirmed

\section{PROPOSED SCHEME}

\section{Whale Optimization Algorithm (WOA)}

Whale Optimization Algorithm (WOA) Whales are fancy, thought-provoking creatures, as adult whales can grow up to $30 \mathrm{~m}$ in length and $180 \mathrm{t}$ in weight which is the most extensive mammals of the world. The giant mammal is made up of 7 different species: Minke, Sei, humpback, right, finback and blue. Whales are regarded predators most of the time. The reason they do not sleep is because they have to breathe from the oceans ' surface. The problem with whales is that they are thought to be highly clever and sense creatures.

Whales in some regions of their brains comparable to normal spindle cells, the Hof and Van Der Gucht report, have prevalent cells. These cells are responsible for human judgement, emotions and social behavior. In other phrases the spindle neurons create U.S. separately from other animals. Whales have a dual cell variety than adults, which is the primary cause of their intelligence. Whale has demonstrated to be thinking, learning, judging, communicating and emotionally even like a human being, but clearly with far less intelligent. Wales (mainly killer whales) were found willing to further create a non-standard speech. The social behavior of whales is another intriguing objective.
They reside in communities or alone. But mostly in communities they are noted. One of the main whales in the horn is the humpback whale. An individual whalebone is kind of like a college bus's size. Some of their types (e.g., killer whales) will live a whole family.

\section{Mathematical model and optimization algorithm}

This chapter initially contains the mathematical model of the prey encircling, the spiral bubble-net feeding strategy and the search for Prey.

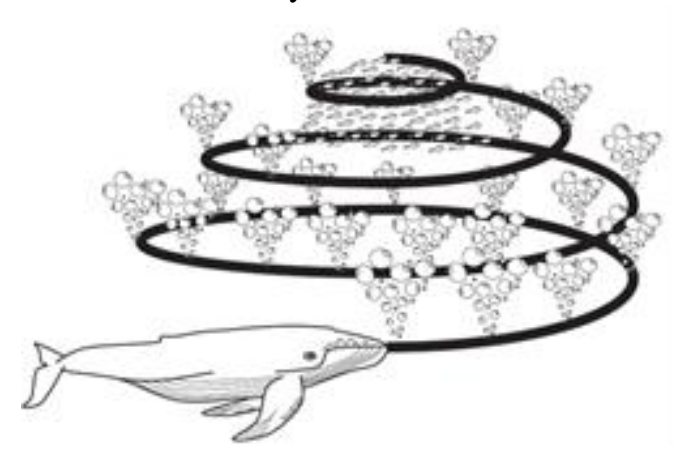

\section{Fig1 . Bubble-net feeding behavior of humpback whales}

\section{System Architecture}

In our proposed system to be working above architecture. Initially preprocessed the input image that is transformed to binary image. The preprocessed image to be used to extract the feature and convolution neural network to be used to train the data based on the feature selection. Then segmentation phase to be completed using WOA algorithm. This algorithm mainly used for to reduce the computational time and increase the accuracy rate.

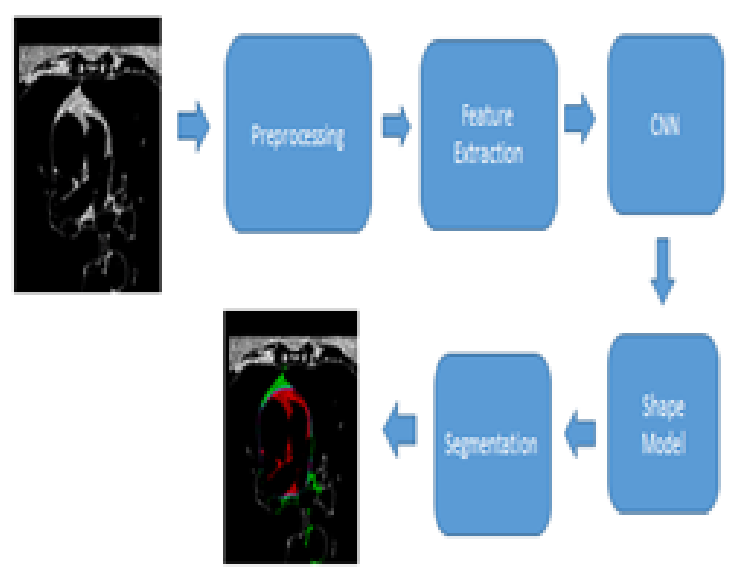

Fig2. Architecture of the system

\section{Encircling prey}

Humpback whales will recognize and encircle the location of the prey. Since the search area's location in the finest style is a priori not better known, the WOA algorithm principle implies that the most effective response for the 
latest candidate is the optimal destination location or nearest. The other search agents will thus attempt to change their roles for the better search agent after it has been established. The following equations show this conduct:

$$
\begin{aligned}
& \vec{D}=\left|\vec{C} \cdot \overrightarrow{X^{*}}(t)-\vec{X}(t)\right| \\
& \vec{X}(t+1)=\overrightarrow{X^{*}}(t)-\vec{A} \cdot \vec{D}
\end{aligned}
$$

Where $\mathrm{t}$ indicates the current iteration, $\mathrm{A}$ and $\mathrm{C}$ are the vector coefficient, $\mathrm{X}=$ is the best solution location vector obtained up-to-date, $X$ is the vector position, || is the complete score and $\bullet$ is element-by-element multiplication. Here it should be pointed out that in each iteration, $\mathrm{X} *$ should be changed if a stronger alternative exists.

The vectors $\mathrm{A}$ and $\mathrm{C}$ can be computed as

$$
\begin{aligned}
& \vec{A}=2 \vec{a} \cdot \vec{r}-\vec{a} \\
& \vec{C}=2 \cdot \vec{r}
\end{aligned}
$$

When a has been reduced linearly from 2 to 0 (both discovery and exploitation stages) during iterations, and $r$ in[ $0,1]$ is a random vector.

Fig. (a) The reason behind $\mathrm{Eq}$ for a $2 \mathrm{D}$ issue is illustrated. The search agent(SA) position $(\mathrm{X}, \mathrm{Y})$ is converted to the present highest record position $(\mathrm{X} *, \mathrm{Y} *)$. The significance of $\mathrm{A}$ and $\mathrm{C}$ vectors can be adjusted at different locations around the finest $\mathrm{SA}$ in relation to the present position. Figure(b) also shows the SA feasible updating position in $3 \mathrm{D}$ space. It should be observed that it is feasible to achieve any position in the search space between the key points displayed in Fig (b) by identifying a Random Vector (r). So Eq. (2.2) enables every SA to renew its present finest solution neighborhood position and simulates the prey's surrounding. The same idea can be applied to a n-dimensional search space and the SA's will navigate around the greatest alternative achieved so far in hypercubes. The humpback whales also attack the preying with a tactic on bubble-net, as stated in the earlier chapter. The following technique is expressed mathematically

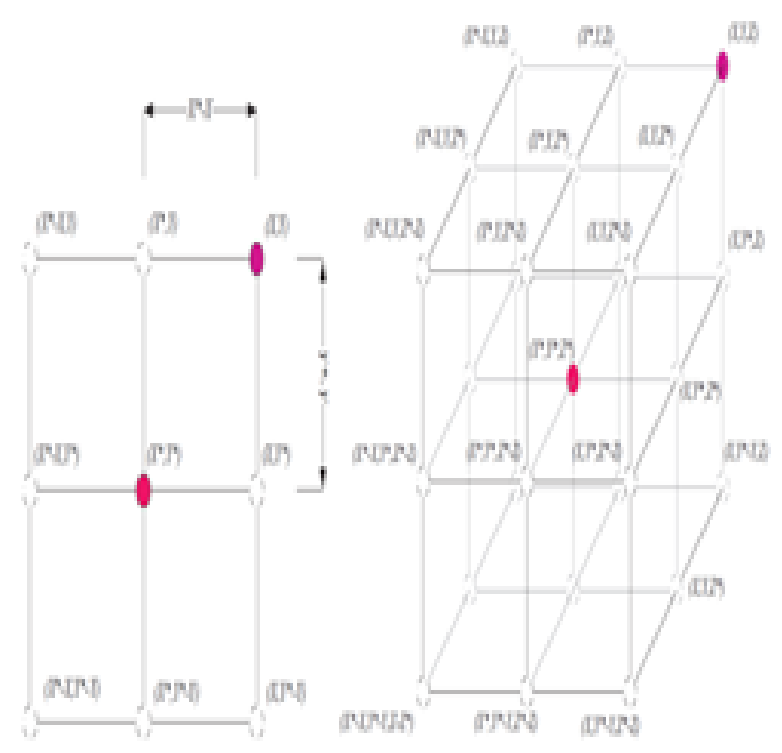

Fig3. 2D and 3D position vectors and their possible next locations ( $X *$ is the best solution obtained so far).

\section{Bubble-net attacking method (exploitation phase)}

Two approaches are designed to model the mathematical bubble-net behavior of humpback whales: 1 Shrinking circular mechanism: This is achieved by reducing the value of an in the Eq. (2.3) Note that A also has a reduced fluctuation variation. In other words, in the interval $[-\mathrm{a}, \mathrm{a}]$, $\mathrm{A}$ is a random value when a function is reduced over the iteration process from 2 to 0 . If the random values in A are set to $[-1,1]$, anywhere between the initial position of the SA and the present finest SA, can describe a new position of SA. Possible (X, Y)-to- $(\mathrm{X} *, \mathrm{Y}-*)$, to be obtained in a $2 \mathrm{D}$ space by $0<=A<=1$.

2 Dl Updating position: this strategy calculates the range between the whale in $(\mathrm{X}, \mathrm{Y})$ and the target in $(\mathrm{X}, \mathrm{Y}, \mathrm{Y})$ first. A circular matrix between the whale and the target is then developed to imitate the helix motion of the humpback whales as follows:

$$
\vec{X}(t+1)=\overrightarrow{D^{\prime}} \cdot e^{b l} \cdot \cos (2 \pi l)+\overrightarrow{X^{*}}(t)
$$

Whale to the target (the finest option so far), $b$ is the limit of the logarithmic curve form definition, 1 is the random number of $[-1,1]$. and from theoretical point of view, WOA can be regarded as a worldwide optimizer since this involves exploration / operation capability and is a element by element multiplication. In addition, the suggested hyper cube system describes a search area in the vicinity of the best alternative and enables other SA's to use the latest record within the domain. The WOA algorithm permits a smooth journey between discovery and exploitation with adaptive variety of the search vector $\mathrm{A}$ : some iterations are devoted to exploring by reducing $A$ (A 1), with the remainder being dedicated to exploitation $(\mathrm{A}<1)$. Remarkably, the WOA only contains two major adjustable internal parameters (A and $\mathrm{C}$ ).

\section{PSEUDO-CODE OF THE WOA ALGORITHM \& RESULTS}

Although the WOA formulation may have included mutation and other evolutionary actions to completely reproduce the activity of humpback whales, we have agreed to reduce the number of heuristics and numerous internal parameters, formulating an incredibly basic version of the WOA algorithm. However, future research can focus on hybridisation with developmental exploration systems 


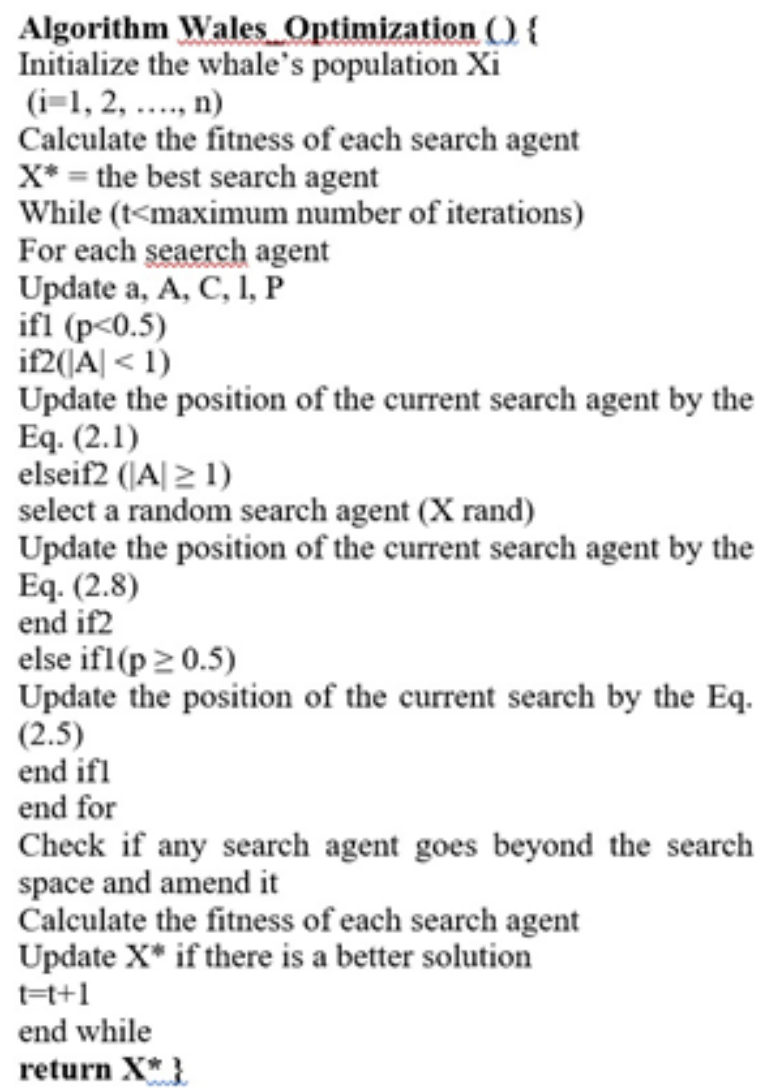

\section{CONCLUSION}

In this system to predict the amount of fat deposited in the layer of pericardium. Further fat to be classified using the tissue identification like us EAT (Epicardial adipose Tissue) and PAT (Paracardial Adipose Tissue) and are differentiated using red and green color. The volume of the fat is presented in the image using volumetric analysis report. The suggested scheme uses fuzzy c-means algorithm and whale optimization algorithm to evaluate fat quantity to enhance prediction of adverse cardiovascular events.

\section{REFERENCES}

[1] A. A. Mahabadi, J. M. Massaro, G. A. Rosito, D. Levy, J. M. Murabito, P. A. Wolf, C. J, O'Donnell, C. $\mathrm{S}$. Fox, and U. Hoffmann, "Association of pericardial fat, intrathoracic fat, and visceral abdominal fat with cardiovascular disease burden: the Framingham Heart Study," European Heart Journal, vol. 30(7), pp. 850$856,2009$.

[2] A. A. Mahabadi, N. Reinsch, N. Lehmann, J. Altenbernd, H. Kalsch, R. M. Seibel, R. Erbel, and S. Mohlenkamp, "Association of pericoronary fat volume with atherosclerotic plaque burden in the underlying coronary artery: a segment analysis," (in eng), Atherosclerosis, vol. 211, no. 1, pp. 195-9, Jul 2010.

[3] B. Tamarappoo, D. Dey, H. Shmilovich, R. Nakazato, H. Gransar, V. Y. Cheng, J. D. Friedman, S. W. Hayes, L. E. Thomson, P. J, Slomka et al., "Increased pericardial fat volume measured from noncontrast CT predicts myocardial ischemia by SPECT," (in eng), JACC Cardiovase Imaging, vol. 3, no. 11, pp. 1104-12, Nov 2010 ,

[4] T. Mazurek, L. Zhang, A. Zalewski, J. D. Mannion, J. T. Diehl, H. Arafat, L. Sarov-Blat, S, O'Brien, E. A Keiper, A. G. Johnson et al., "Human Epicardial Adipose Tissue Is a Source of Inflammatory Mediators," Circulation, vol. 108, pp. 2460-2466, 2003.

[5] M. Shimabukuro, Y. Hirata, M. Tabata, M. Dagyasumberel, H. Sato, H. Kurobe, D. Fukuda, T. Soeki, T. Kitagawa, S. Takanashi et al., "Epicardial adipose tissue volume and adipocytokine imbalance are strongly linked to human coronary atherosclerosis," (in eng), Arterioscler Thromb Vasc Biol, vol, 33, no. 5, pp. 1077-84. Mav 2013.

[6] A. H. Talman, P. J, Psaltis, J. D. Cameron, I. T. Meredith, S. K. Seneviratne, and D. T. L. Wong, "Epicardial adipose tissue: far more than a fat depot," Cardiovascular Diagnosis and Therapy; vol. 4, no. 6 , pp. 416-429, 2014

[7] V. Y. Cheng, Dey, D, Tamarappoo, B.K., Nakazato R. Gransar, H, Miranda-Peats R, Ramesh A, Wong N.D. Shaw L.J., Slomka P.J., Berman D.S., "Pericardial fat burden on ECG-gated noncontrast CT in asymptomatic patients who subsequently experience adverse cardiovascular events on 4-year follow-up: A casecontrol study," Journal of $\mathrm{Am}$ Coll Cardiol Cardiovascular Imaging pp. 352-360.

[8] A. A. Mahabadi, J. M. Massaro, G. A. Rosito, D. Levy, J. M. Murabito, P. A. Wolf, C. J. O'Donnell, C. S. Fox, and U. Hoffmann, "Association of pericardial fat, intrathoracic fat, and visceral abdominal fat with cardiovascular disease burden: the Framingham Hear Study," (in eng), Eur Heart J, Journal Article vol. 30 , no. 7, pp.850-6, Apr 2009.

[9] A. A. Mahabadi, N. Lehmann, H. Kalsch, T. Robens, M. Bauer, I. Dykun, T. Budde, S. Moebus, K. H Jockel, R. Erbel et al., "Association of epicardial adipose tissue with progression of coronary artery calcification is more pronounced in the early phase of atherosclerosis: results from the Heinz Nixdorf recall study," (in eng) JACC Cardiovasc Imaging, Journal Article Multicenter Study Research Support, Non-U.S Gov't vol. 7, no. 9, pp. 909-16, Sep 2014.

[10] A. A. Mahabadi, M. H. Berg, N. Lehmann, H. Kalsch. M. Bauer, K. Kara, N. Dragano, S. Moebus, K. H. Jockel, R. Erbel et al., "Association of epicardial fat with cardiovascular risk factors and incident myocardial 
infarction in the general population: the Heinz Nixdorf Recall Study," (in eng), $J$ Am Coll Cardiol, Journal Article Research Support, Non-U.S. Gov't vol. 61 , no. 13, pp. 1388-95, Apr 22013.

[11] R. M. Abazid, O. A. Smettei, M. O. Kattea, S. Sayed, H. Saggah, A. M. Widyan, and M. P. Opolski, "Relation Between Epicardial Fat and Subclinical Atherosclerosis in Asymptomatic Individuals," Joumal of Thoracic Imaging 2017.

[12] A. S. Agatston, W. R. Janowitz, F, J. Hildner, N, R. Zusmer, M. Viamonte, and R. Detrano, "Quantification of coronary artery calcium using ultrafast computed tomography," Journal of the American College of Cardiology, vol. 15, no. 4, pp. 827-832, 1990.

[13] T. Q. Callister, B. Cooil, S. P. Raya, N. J. Lippolis, D. J. Russo, and P. Raggi, "Coronary artery disease: improved reproducibility of calcium scoring with an electron-beam CT volumetric method," Radiology, vol. 208, no. 3, pp. 807-814, 1998.

[14] D. Dey, Y. Suzuki, S. Suzuki, M. Ohba, P. J. Slomka. D. Polk, L. J. Shaw, and D. S. Berman, "Automated quantitation of pericardiac fat from noncontrast CT," Investigative radiology: vol. 43 , no. 2 , pp. 145-153, 2008.

[15] Y. LeCun, Y. Bengio, and G. Hinton, "Deep learning." Nature, vol. 521, no. 7553, pp. 436-444, 2015.

[16] D. Dey, N. D. Wong, B. Tamarappog, R. Nakazato, H. Gransar, V. Y. Cheng, A. Ramesh, I. Kakadiaris, G. Germano, and P. J. Slomka, "Computer-aided noncontrast CT-based quantification of pericardial and thoracic fat and their associations with coronary calcium and metabolic syndrome," Atherosclerosis, vol. 209, no. 1. pp. 136-141, 2010.

[17] V. Y. Cheng, D. Dey, B. Tamarappoo, R. Nakazato, H. Gransar, R. Miranda-Peats, A. Ramesh, N. D. Wong. L. J. Shaw, and P. J. Slomka, "Pericardial fat burden on ECG-gated noncontrast CT in asymptomatic patients who subsequently experience adverse cardiovascular events," JACC: Cardiovascular Imaging, vol. 3, no. 4, pp. $352-360,2010$.

[18] R. Nakazato, H. Shmilovich, B. K. Tamarappoo, V. Y. Cheng. P. J. Slomka. D. S. Berman, and D. Dey, "Interscan reproducibility of computer-aided epicardial and thoracic fat measurement from noncontrast cardiac CT," Journal of cardiovascular computed tomography, vol. 5, no. 3, pp. 172-179, 2011.

[19] J. G. Barbosa, B. Figueiredo, N. Bettencourt, and J. M. R. Tavares, "Towards automatic quantification of the epicardial fat in non-contrasted CT images," Computer methods in biomechanics and biomedical engineering. vol. 14, no. 10, pp. 905-914, 2011.

[20] X. Ding, D. Terzopoulos, M. Diaz-Zamudio, D. S. Berman, P. J. Slomka, and D. Dey, "Automated epicardial fat volume quantification from non-contrast CT," in SPIE Medical Imaging, 2014, pp. 90340I90340I-6: International Society for Optics and Photonics:

[21] X. Ding. D. Terzopoulos, M. Diaz-Zamudio, D. S. Berman, P. J. Slomka, and D. Dey, "Automated pericardium delineation and epicardial fat volume quantification from noncontrast CT," Medical physics, vol. 42, no. 9, pp. 5015-5026, 2015 . 